\title{
PRIMER REGISTRO DE LA MOSCA BLANCA DE LOS CEREALES ALEUROCYBOTUS OCCIDUUS RUSSELL (HEMIPTERA: ALEYRODIDAE) Y SU IMPACTO POTENCIAL COMO PLAGA DE GRAMINEAS EN EL NORTE DE SINALOA
}

\author{
Guadalupe VeJAR-Cota ${ }^{1}$, Laura Delia ORTEga-AREnAS ${ }^{2}$ \\ y Vicente Emilio CARAPIA-RUIZ 3 \\ ${ }^{1}$ Cía. Azucarera de Los Mochis, S.A de C.V. Prol. Vicente Guerrero s/n sur. Los Mochis, Sinaloa, \\ México, MÉXICO. (vejargpe@hotmail.com). \\ 2 Programa en Entomología y Acarología. Campus Montecillo. Colegio de Postgraduados. 56230, \\ Montecillo, Estado de México. MÉXICO. (ladeorar@colpos.mx). \\ 3 Campus Oriente. Universidad Autónoma del Estado de Morelos.62742, Cuautla, Morelos, \\ MÉXICO. (vcarapia@hotmail.com).
}

Vejar Cota, G., L. D. Ortega-Arenas y V. E. Carapia-Ruiz. 2009. First record of the cereal whitefly Aleurocybotus occiduus Russell (Hemiptera: Aleyrodidae) and its potential impact as a cereal pest in Northern Sinaloa. Acta Zool. Mex. (n. s.) 25(1): 33-48.

RESUMEN. Se identifica la mosca blanca de los cereales Aleurocybotus occiduus Russell (Hemiptera: Aleyrodidae) a partir de especímenes procedentes de Guasave, Sinaloa, México. Se identificaron 10 especies vegetales, entre poáceas y ciperáceas, como hospederas de $A$. occiduus. Sin embargo, sólo en ocho de ellas (seis silvestres y dos cultivadas) completa su ciclo de vida. Este aleiródido se colectó en todos los sitios cercanos a las ciudades de Los Mochis y Guasave, Sinaloa; no obstante, hasta ahora no ha ocasionado daños importantes a los cultivos debido en parte a la presencia de parasitoides Encarsia spp. y Eretmocerus spp. (Aphelinidae) obtenidos en muestras de A. occiduus. Este es el primer registro de la especie en el estado de Sinaloa, por lo que mas atención debe darse a este insecto debido al riesgo potencial que tiene para atacar cereales en la región.

Palabras clave: Moscas blancas, Aleurocybotus, Poaceae, hospederas, distribución, enemigos naturales.

Vejar Cota, G., L. D. Ortega-Arenas \& V. E. Carapia-Ruiz. 2009. First record of the cereal whitefly Aleurocybotus occiduus Russell (Hemiptera: Aleyrodidae) and its potential impact as a cereal pest in Northern Sinaloa. Acta Zool. Mex. (n. s.) 25(1): 33-48.

ABSTRACT. Aleurocybotus occiduus Russell (Hemiptera: Aleyrodidae) was identified from specimens collected at Guasave, Sinaloa State, Mexico. Ten plant species, all among Poaceae and Cyperaceae, were identified as hosts of this insect at Guasave fields. However, A. occiduus was able to complete its life cycle only in eight of those hosts, six wild and two domesticated species. The aleyrodid was collected from sites close to cities Los Mochis and Guasave, Sinaloa, nevertheless it seems that it is not causing important damages to the cultivated plants in that region, the parasitoids Encarsia spp. and Eretmocerus spp.(Aphelinidae) that have been recovered from A. occiduus samples might have something to do with. This constitutes the first record of this species at Sinaloa 
state, and more attention should be given to this insect because the potential risk for attacking cereals in that region.

Key words: Whiteflies, Aleurocybotus, Poaceae, hosts, distribution, natural enemies.

\section{INTRODUCCIÓN}

Las moscas blancas (Hemiptera: Aleyrodidae) constantemente son incluidas en las listas de plagas en México y el mundo (Ortega 1999, Hodges \&Evans 2005). El impacto económico causado por el complejo moscas blancas-virosis, ha provocado en México situaciones de verdadera emergencia, a grado tal que se han tenido que destruir miles de hectáreas de cultivos diversos, debido a la mala calidad, manchado y bajos rendimientos (Ortega 1999).

A nivel mundial se citan 1,556 especies de moscas blancas, incluidas en 161 géneros (Martín \& Mound 2007). En México, de las 67 especies reportadas, sólo son reconocidas de importancia económica a Bemisia tabaci (Gennadius), B. argentifolii Bellows \& Perring y Trialeurodes vaporariorum (Westwood), las cuales ocurren principalmente en cultivos de hoja ancha como melón, sandía, chile, tomate, algodonero, frijol, col y cítricos (Ortega 1999), aunque los géneros Agrostaleyrodes, Aleurocanthus, Aleurocybotus, Aleurolobus, Aleuroplatus, Aleurotrachelus, Aleurotulus, Bemisia, Paraleyrodes, Trialeurodes y Vasdavidius cuentan con representantes que atacan plantas de hoja angosta (Poaceae) (Evans 2007).

En diciembre de 2006 se registro una fuerte incidencia de una especie de mosca blanca en sorgo, caña de azúcar, maíz y algunas malezas en el ejido Adolfo Ruíz Cortinez, Guasave, Sinaloa, por lo que los productores solicitaron el apoyo gubernamental para el control de la plaga y a los autores de este escrito la determinación de la mosca que estaba dañando sus cultivos. La especie fue determinada como Aleurocybotus occiduus Russell, siendo este el primer registro para Sinaloa y México.

La mosca blanca de los cereales (MBC) Aleurocybotus occiduus Russell (Hemiptera: Aleyrodidae) es una especie neotropical, la cual se encuentra distribuida en EUA (Arizona, California, Florida y Hawai), Perú (Martín 2004, Evans 2007), El Salvador (Serrano et al. 2006) y ahora en Sinaloa, México. El insecto ocurre en alrededor de 23 especies de plantas silvestres y cultivadas (poáceas y ciperáceas) en América (Martín 2004, Serrano et al. 2006, Evans 2007). Los daños causados por $A$. occiduus son similares a otros aleiródidos. Las ninfas y adultos causan marchitez, clorosis y achaparramiento, por succión de savia, lo cual se traduce en perdida de calidad y rendimiento del cultivo o la caída prematura de las hojas, o muerte prematura, en infestaciones severas, por la excesiva proliferación de hongos "fumagina" sobre la mielecilla que excretan. No se tienen reportes a la fecha que indiquen que $A$. occiduus pueda transmitir virus (Poinar 1965). 
Serrano et al. (2004) anotan que en Chalatenango, El Salvador, A. occiduus se presentó como una plaga importante en arroz (Oryza sativa) llegando a causar la perdida total de la cosecha. Después del arroz, la plaga comenzó a reproducirse en abundancia en plantas jóvenes de maicillo (Sorghum bicolor), perdiéndose del 80$100 \%$ de la cosecha esperada. Una situación similar fue reportada por Heinrichs \& Barrion (2004) en Fayane y NDiane, Senegal y en Nigeria, África, donde la presencia de $A$. indicus causó la destrucción total de las plantaciones de arroz.

Por lo antes expuesto y debido a la fuerte incidencia de $A$. occiduus observada en gramíneas en Sinaloa y a que la especie se registra por primera vez en México, se plantearon como objetivos: a) realizar la descripción morfológica de la especie; b) realizar un listado de plantas silvestres y cultivadas hospederas de la mosca; d) conocer el área de distribución de la mosca en el norte de Sinaloa; e) realizar observaciones sobre la biología y comportamiento de $A$. occiduus en las condiciones del norte de Sinaloa; f) identificar el complejo de enemigos naturales asociados a la mosca; g) revisar su situación como plaga potencial para las gramíneas cultivadas en el norte de Sinaloa.

\section{MATERIAL Y MÉTODOS}

Sitio de estudio. El estudio se realizó en diferentes localidades del norte de Sinaloa (Fig. 1), que comprenden a los Distritos de Los Mochis (25 $48^{\prime}$ Latitud Norte, $109^{\circ} 00^{\prime}$ Latitud Oeste, $\left.10 \mathrm{msnm}\right)$ y Guasave (25 $34^{\prime}$ Latitud Norte, $108^{\circ} 28^{\prime}$ Latitud Oeste, $10 \mathrm{msnm}$ ), que corresponden a los valles agrícolas de El Fuerte y Guasave, respectivamente.

La temperatura media anual que prevalece en esta región es de $25^{\circ} \mathrm{C}$ con máximas de $43^{\circ} \mathrm{C}$, en los meses de agosto y septiembre y mínimas de $2^{\circ} \mathrm{C}$ registradas en enero. La precipitación media anual es de $352 \mathrm{~mm}$, y la mayor parte se registra en los meses de julio a septiembre. El clima es cálido, seco estepario (tipo desértico) en el verano, mientras que en el invierno es moderadamente frío en los meses de noviembre a enero y templado a partir de febrero (INEGI 2001, Anónimo 2003).

Colecta de material biológico y plantas hospederas. Los muestreos se realizaron en dos periodos de acuerdo a la aparición de la plaga. De diciembre 2006 a abril 2007 (Periodo 1) y de mayo a julio 2007 (Periodo 2). En ambos casos el muestreo consistió en contabilizar el número de individuos (huevos, ninfas, pupas o adultos) en cada punto de muestreo, realizado en 15 minutos de búsqueda. En cada sitio se revisaron las hojas inferiores (previa observación del comportamiento del insecto) de las diferentes especies de plantas silvestres y cultivadas posibles hospederos de la mosca, localizadas en orillas de cultivos, caminos, drenes y canales.

Periodo 1. La primera detección de A. occiduus se ubicó $2 \mathrm{~km}$ al sur del Ejido Adolfo Ruíz Cortínez por la carretera internacional No 15 México-Nogales (31 km al sur de Los Mochis). Conforme se realizaron las observaciones sobre los hospederos 


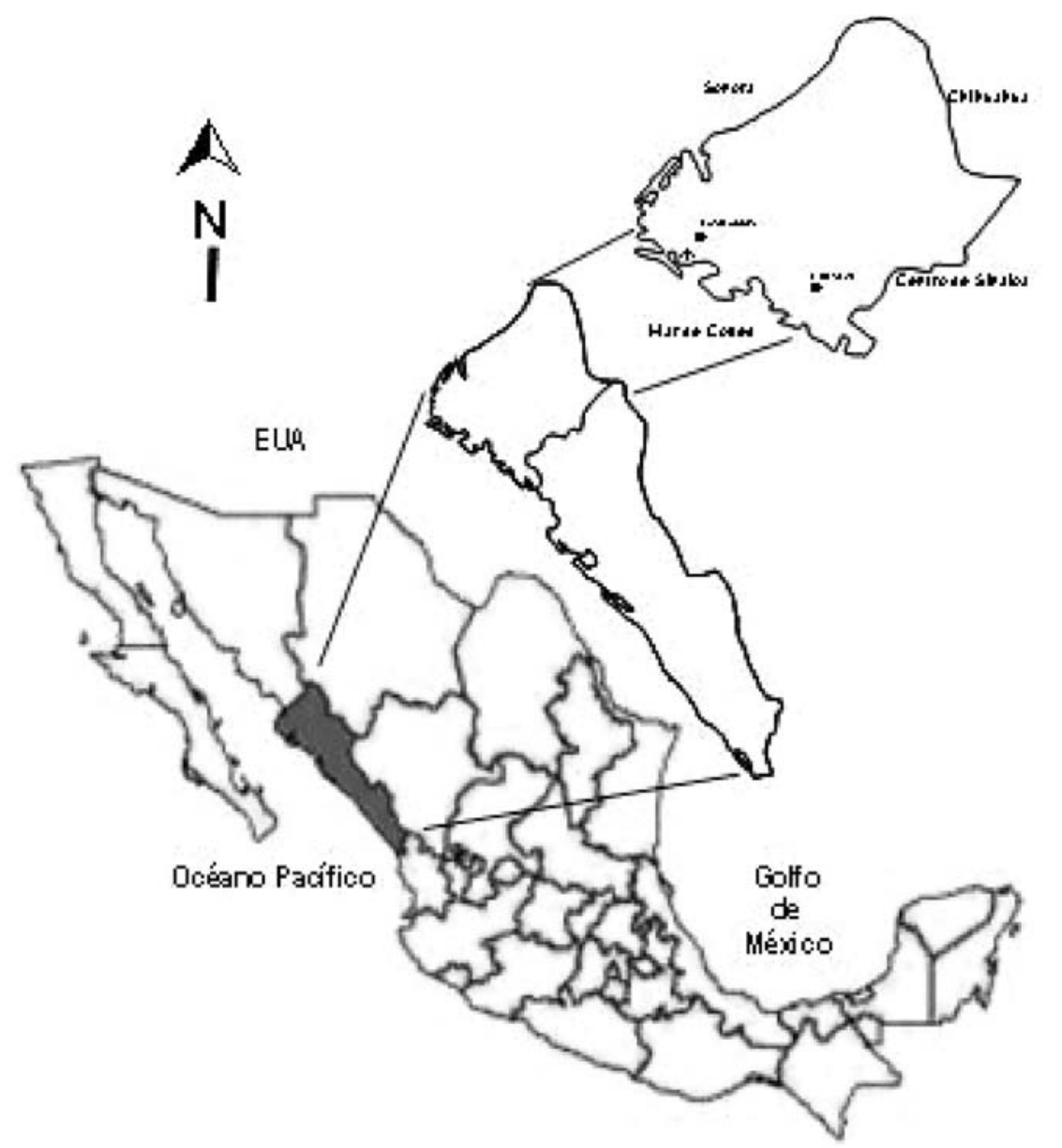

Figura 1. Ubicación geográfica del sitio de estudio de la mosquita blanca de los cereales A. occiduus.

preferenciales de la mosca blanca, se disminuyeron las hospederas potenciales a revisar. El muestreo concluyó hasta donde se dejaron de obtener especímenes. Además, y con la finalidad de conocer en forma amplia la distribución de la plaga se realizaron recorridos exploratorios en diferentes localidades de los valles de El Fuerte y Guasave, tanto en cultivos en pie como en malezas.

Periodo 2. Durante este periodo se inspeccionaron los sitios revisados en el primer periodo y se agregaron otros más lejanos para delimitar la dispersión de la MBC.

De cada especie vegetal, que albergaba a las moscas, se tomaron dos ejemplares, se prensaron y se les asignó una clave de colecta en la que se anotó la fecha, localidad y algunos datos sobre abundancia y características útiles para la identificación. 
Posteriormente, los ejemplares se llevaron al laboratorio para someterlos a secado y dejarlos listos para su determinación, la cual fue realizada por Ing. Roberto Bañuelos Gijón (Cía. Azucarera de Los Mochis, S.A. de C. V., Especialista en malezas). Asimismo, los adultos y ninfas de mosca blanca por especie vegetal se conservaron en alcohol al 70\%, para corroborar su determinación la cual fue realizada por la Dra. Laura Delia Ortega Arenas (Programa en Entomología, Colegio de Postgraduados) y el Dr. Vicente Emilio Carapia Ruiz (Universidad Autónoma del Estado de Morelos).

Descripción morfológica. Las "pupas", exuvias y adultos para realizar la descripción de la especie fueron analizadas en un microscopio estereoscópico a 40 aumentos antes de someterlas al proceso de montaje en laminillas descrito por Martin (1987).

Las preparaciones se analizaron en un microscopio compuesto a 100, 400 y 1000 aumentos, se realizaron mediciones con un micrómetro con aproximación máxima de $1 \mu \mathrm{m}$ y se tomaron fotografías con un fotomicroscopio III Carl Zeiss con cámara Paxcam 3. La descripción se realizó usando la terminología detallada por Russell (1964), Martin (1987), Ortega (1995) y Hodges \& Evans (2005). Los ejemplares estudiados se depositaron en las colecciones del Colegio de Postgraduados, Campus Montecillo y la Universidad Autónoma del Estado de Morelos.

Biología y Comportamiento. Durante los muestreos, se realizaron además anotaciones sobre biología (apariencia, estados de desarrollo), hábitos, y comportamiento de los especímenes colectados.

Abundancia y diversidad de enemigos naturales. Para determinar la abundancia y diversidad de enemigos naturales de la MBC, las hojas o porciones de foliolos de las diferentes hospederas que contenían ninfas parasitadas o mostraban evidencia de presencia de algún enemigo natural, se colocaron en cajas Petri con algodón húmedo. Cada día se revisaban para determinar la emergencia y los enemigos emergidos se clasificaron por apariencia. Se efectuaron montajes tanto de los depredadores como de parasitoides, estos últimos en laminillas, para su determinación taxonómica, la cual fue realizada por M.C Guadalupe Vejar (Cía. Azucarera de Los Mochis, S.A de C.V.).

\section{RESULTADOS}

Descripción morfológica de Aleurocybotus occiduus Russell 1964. Especímenes in vivo. Los adultos blancos-grisáceos en apariencia que semejan diminutas palomillas con las alas dispuestas en forma de tejado y cuerpo a menudo cubierto por un polvillo ceroso. La hembra mide en promedio $1.2 \mathrm{~mm}$ de largo, con una expansión alar de $1.3 \mathrm{~mm}$; mientras que el macho es más pequeño y mide $0.7 \mathrm{~mm}$ de largo y expansión alar de $0.8 \mathrm{~mm}$ (Fig. 2a). Los huevos son alargados y curveados en el centro y son colocados de manera horizontal y adheridos a los estomas de las hojas por un pequeño pedicelo. Recién depositados son color blanco y conforme maduran 
Vejar-Cota et al. Mosca blanca de los cereales en Sinaloa
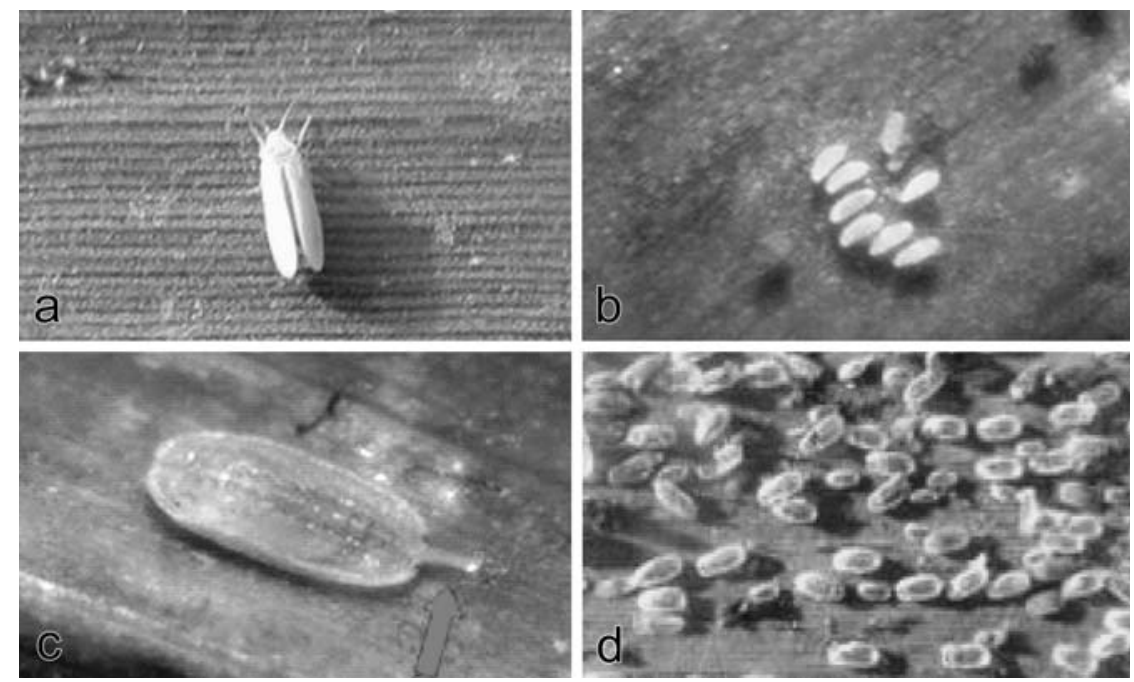

Figura 2. Fases de desarrollo de A. occiduus: a) adulto, b) huevos en maíz Zea mays, c) ninfa con detalle de filamentos caudales cerosos, d) ninfas en altas densidades en caña de azúcar $S$. officinarum.

oscuros con el corion brilloso. Miden alrededor de $0.3 \mathrm{~mm}$ de largo por $0.1 \mathrm{~mm}$ de ancho (Fig. 2b). La hembra al ovipositar deja una capa de polvo blanquecino alrededor de los huevos. Las ninfas tienen el cuerpo oval aplanado, semitransparente, color amarillento, 2-3 veces más largo que ancho, y presentan un par de filamentos cerosos en la región caudal (Fig. 2c). Este estado de desarrollo pasa por cuatro instares. La ninfa en su última etapa mide en promedio 1.1-1.4 $\mathrm{mm}$ de largo por 0.5$0.7 \mathrm{~mm}$ de ancho. Las ninfas y adultos ocurren en densas colonias que a menudo cubren completamente el envés de las hojas (Fig. 2d).

Especímenes montados en laminillas. Cubierta pupal: Color amarillo pálido, a menudo con tonalidades rojas, marrón o incoloras de forma elíptica o subelíptica. Cera transparente incolora en el dorso y vientre, cera blanca traslucida circundando la parte ventral de la ninfa madura. Los especímenes analizados varían de aproximadamente 1.1-1.4 mm de largo por 0.5-0.7 $\mathrm{mm}$ de ancho. Estados inmaduros encontrados en pastos, aunque en los últimos años se han encontrado en arroz, maíz y caña de azúcar, comúnmente formando agregados. Margen elongado, oval, simétrico y dentado. Seta anterior y posterior presente o anterior no observada. Poros traqueales ausentes (Fig. 3a).

Dorso: Ligeramente esculpido, ligeramente esclerosado, semiaplanado y con un raquis medio en el abdomen. Depresiones medianas y bolsas presentes (Fig. 3b). Ocho segmentos presentes en la parte mediana del abdomen. Suturas transversa y 

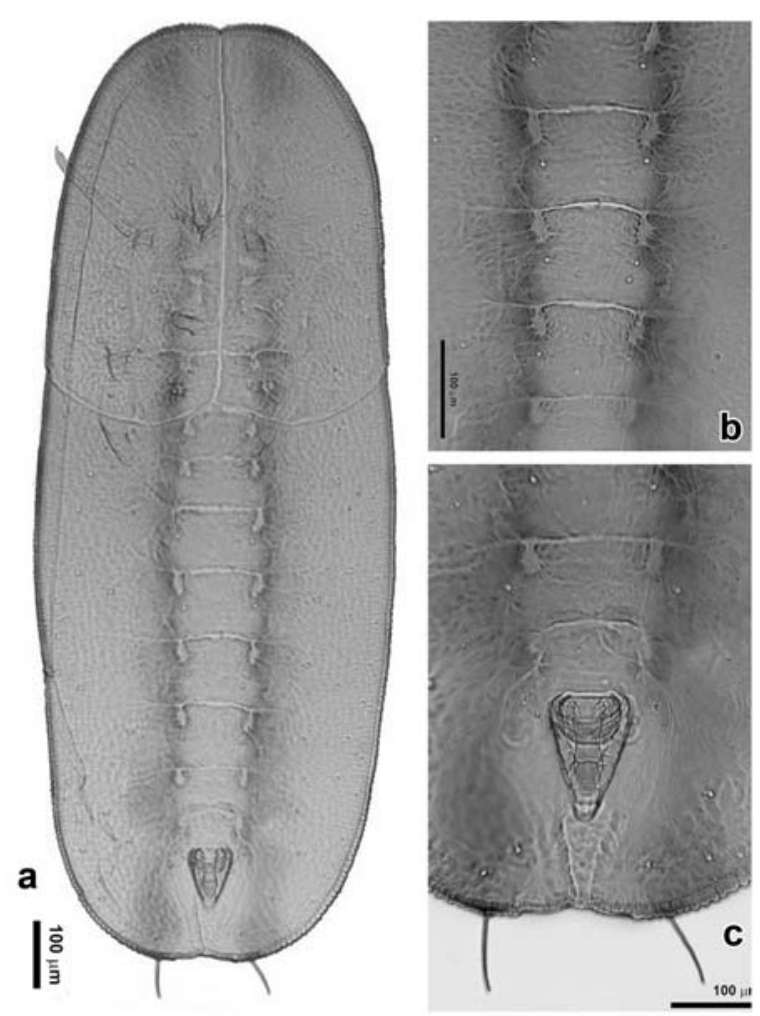

Figura 3. Aleurocybotus occiduus Russell. a) cubierta pupal (vista dorsal), b) depresiones en el área dorsal media, c) orificio vasiforme. Barra $=100 \mu \mathrm{m}$.

longitudinal de la muda alcanzan el margen del cuerpo, sutura transversa de la muda curvada ligeramente hacia atrás de su punto medio, recurvado para alcanzar el margen del cuerpo ligeramente anterior al punto opuesto de su punto medio. Poros del disco y poretes asociados, aproximadamente 5 ó 6 pares por segmento. Seta cefálica presente, primera seta abdominal presente; octava seta abdominal y seta caudal presentes aunque el par de estas últimas es común que se desprendan en el proceso de montaje debido a su tamaño relativamente grande; usualmente un par de setas submarginales en cada segmento del tórax y en tres o mas segmentos abdominales. Setas diminutas o bases de setas subdorsales o submedianas algunas veces presentes en el tórax y abdomen (Fig. 3a). Orificio vasiforme semitriangular, extremo posterior cerrado. Opérculo más ancho que largo y recurvado anteriormente en su parte posterior. Lígula espatulada, extendiéndose más allá del opérculo, contenida en el orificio. Surco caudal extendiéndose del orificio al margen posterior del cuerpo (Fig.3c). 
Vientre: incoloro, membranoso. Aplanado en ninfas jóvenes, hinchado en ninfas maduras. Glándulas de ceras submarginales presentes. Segmento distal del rostro tan ancho como largo, con dos o tres pares de setas diminutas. Antenas localizadas en la parte lateral de las patas, mas largas en el macho que en la hembra, extendiéndose hasta o mas allá del extremo posterior de las patas anteriores, terminando en un proceso en forma de dedo. Sacos adhesivos presentes. Pliegues traqueales ausentes. Espiráculo anterior torácico y posterior abdominal presente. Pata media y anterior algunas veces reducida. Setas ventrales abdominales presentes (Fig. 3a).

Adultos. Cuerpo delgado amarillo pálido o el dorso teñido de marrón o rojo. Cabeza: Cada ojo compuesto dividido por un espacio estrecho; facetas casi idénticas en tamaño y forma. Un ocelo adyacente al margen de la parte superior de cada ojo compuesto. Segmento distal del rostro tan ancho como largo, con dos pares de setas diminutas. Segmento antenal VII mas largo que los segmentos III-VI combinados en machos, mas corto que los segmentos III-VI combinado en hembras, terminando en una delgada membrana con dos filamentos delgados en el macho, un filamento delgado y una seta sensorial en la hembra; uno o mas de los segmentos III-VI con una o mas sensorias.

Tórax: Alas pálidas, sector radial presente. Patas con coxa, trocánter, fémur, tibia y tarso de dos segmentos; tibia posterior con una hilera de setas; con una uña lisa normal y un apéndice tarsal en forma de uña, este con apariencia de protuberancia con pequeñas espínulas, una seta delgada en el margen; sin un paronychium típico.

Abdomen: Área alrededor del orificio vasiforme ligeramente esclerosada. Orificio vasiforme tan ancho como largo, extremo posterior cerrado. Opérculo cuadrado o más ancho que largo. Lígula espatulada, extendiéndose más allá del orificio. Válvulas del ovipositor delgadas casi puntiagudas en el ápice. Claspers del macho más delgados, puntiagudos en el ápice, con pocas setas delgadas. Aedeago adelgazándose de la base al ápice.

Comentarios. La especie Aleurocybotus occiduus Russell es similar a Aleurocybotus graminicola (Quaintance \& Baker 1914) pero son claramente diferenciables debido a que $A$. occiduus es marcadamente mas larga, y tiene las depresiones abdominales medianas mas largas que anchas, mientras que en $A$. graminicola son tan o más anchas que largas. El género Aleurocybotus Quaintance y Baker incluye actualmente tres especies, las dos especies mencionadas más Aleurocybotus cereus del continente americano (Martin 2004), mientras que las especies del hemisferio oriental que anteriormente se incluían en este género, Russell (2000) las ubicó en el género Vasdavidius.

Plantas hospederas. Se revisaron en total 17 plantas silvestres y tres cultivadas en el área con presencia de $A$. occiduus (Cuadro 1). En 10 de las especies se detectó la presencia de $A$. occiduus, sin embargo, en dos de ellas la mosca no pudo completar su ciclo biológico y sólo se encontraron huevos y ninfas de los primeros ínstares. En 
las restantes 10 especies (seis de hoja angosta y cuatro de hoja ancha) no se observó colonización de moscas lo cual hace suponer que dichas plantas no son aptas para el desarrollo de la MBC.

A la fecha sólo se han detectado seis hospederas silvestres: Sorghum halepense, Cenchrus ciliaris, Cynodon dactylon, Chloris gayana, Echinochloa colonum (poáceas) y Cyperus rotundus (ciperácea) y dos cultivadas: $S$. vulgare y Saccharum officinarum (Fig. 4) donde la MBC completa su ciclo de vida (Cuadro 1). Dichanthium aristatum (Poiret) se cita por primera vez como hospedero potencial de este aleiródido.
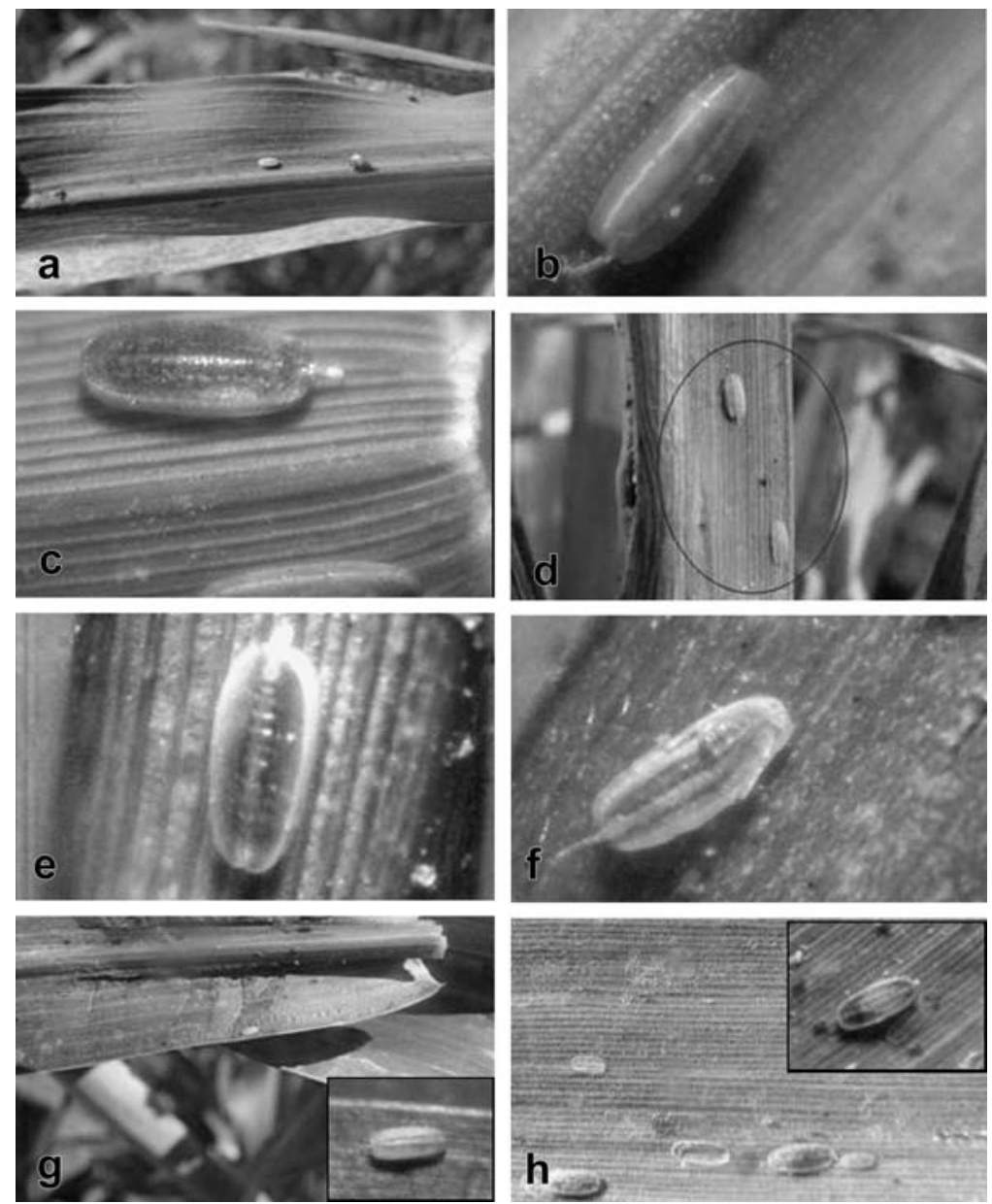

Figura 4. Plantas silvestres y cultivadas donde se reproduce la mosca blanca de los cereales en el norte de Sinaloa. a) S. halepense, b) C. ciliaris, c) C. dactylon, d) C. gayana, e) C. rotundus, f) E. colonum, g) $S$. vulgare, y h) $S$. officinarum. 
Vejar-Cota et al. Mosca blanca de los cereales en Sinaloa

Cuadro 1. Plantas silvestres y cultivadas no hospederas y hospederas de la mosca blanca de los cereales A. occiduus, en el Norte de Sinaloa.

\begin{tabular}{|c|c|c|c|c|c|}
\hline $\begin{array}{l}\begin{array}{l}\text { Nombre } \\
\text { científico }\end{array} \\
\end{array}$ & $\begin{array}{l}\text { Nombre } \\
\text { común }\end{array}$ & Familia & $\begin{array}{l}\text { Presencia de } \\
\text { A. occiduus }\end{array}$ & Aceptación* & Referencias \\
\hline \multicolumn{6}{|l|}{ Plantas silvestres } \\
\hline \multicolumn{6}{|l|}{ Hoja angos ta } \\
\hline Avena fatua $\mathrm{L}$. & $\begin{array}{l}\text { Avena } \\
\text { silvestre }\end{array}$ & Poaceae & & $\mathrm{NC}$ & \multirow{4}{*}{$\begin{array}{l}\text { Martín 2004, Evans } 2007 \\
\text { Russell 1964, Martín } \\
\text { 2004, Evans 2007 } \\
\text { Russell 1964, Poinar } \\
\text { 1965, Martín 2004, Evans } \\
2007\end{array}$} \\
\hline Cenchrus ciliaris $\mathrm{L}$. & Zacate Buffel & Poaceae & $\mathrm{X}$ & $\mathrm{CC}$ & \\
\hline Chloris gayana Kunth. & $\begin{array}{l}\text { Zacate } \\
\text { Rhodes }\end{array}$ & Poaceae & $\mathrm{x}$ & $\mathrm{CC}$ & \\
\hline $\begin{array}{l}\text { Cynodon dactylon (L.) } \\
\text { Pers. }\end{array}$ & $\begin{array}{l}\text { Zacate } \\
\text { Grama }\end{array}$ & Poaceae & $\mathrm{x}$ & $\mathrm{CC}$ & \\
\hline Cyperus rotundus $\mathrm{L}$. & Coquillo & Cyperaceae & $\mathrm{x}$ & $\mathrm{CC}$ & $\begin{array}{l}\text { Russell 1964, Poinar } \\
\text { 1965, Martín 2004, } \\
\text { Serrano et al. 2006, Evans } \\
2007\end{array}$ \\
\hline $\begin{array}{l}\text { Dichanthium } \\
\text { aristatum(Poiret) }\end{array}$ & $\begin{array}{l}\text { Zacate } \\
\text { Angletón }\end{array}$ & Poaceae & $\mathrm{X}$ & $\mathrm{CI}$ & \multirow[b]{2}{*}{ Serrano et al. 2006} \\
\hline $\begin{array}{l}\text { Echinochloa colonum } \\
\text { (L.) Link }\end{array}$ & Zacate pinto & Poaceae & $\mathrm{X}$ & $\mathrm{CC}$ & \\
\hline $\begin{array}{l}\text { Panicum purpurascens } \\
\text { Raddi }\end{array}$ & $\begin{array}{l}\text { Zacate } \\
\text { carricillo }\end{array}$ & Poaceae & & $\mathrm{NC}$ & \multirow{6}{*}{$\begin{array}{l}\text { Russell 1964, Martín } \\
\text { 2004, Evans } 2007\end{array}$} \\
\hline Phalaris minor Retz. & Alpistillo & Poaceae & & $\mathrm{NC}$ & \\
\hline $\begin{array}{l}\text { Phragmites australis } \\
\text { (Cav.) }\end{array}$ & Carrizo & Poaceae & & $\mathrm{NC}$ & \\
\hline $\begin{array}{l}\text { Sorghum helepense } \\
\text { (L.) Pers. }\end{array}$ & $\begin{array}{l}\text { Zacate } \\
\text { Jhons on }\end{array}$ & Poaceae & $\mathrm{x}$ & $\mathrm{CC}$ & \\
\hline Typha an gustifolia $\mathrm{L}$. & Tule & Typhaceae & & $\mathrm{NC}$ & \\
\hline $\begin{array}{l}\text { Urochloa plantaginea } \\
\text { (Link.) RD. Webster }\end{array}$ & $\begin{array}{l}\text { Zacate } \\
\text { horquetilla }\end{array}$ & Poaceae & & $\mathrm{NC}$ & \\
\hline \multicolumn{6}{|l|}{ Hoja ancha } \\
\hline $\begin{array}{l}\text { Amaranthus spinosus } \\
\text { L. }\end{array}$ & $\begin{array}{l}\text { Bledo } \\
\text { colorado }\end{array}$ & Amaranthaceae & & $\mathrm{NC}$ & \\
\hline Helianthus annuus L. & Girasol & Asteraceae & & $\mathrm{NC}$ & \\
\hline Ricinus communis $\mathrm{L}$. & Higuerilla & Euphorbiaceae & & $\mathrm{NC}$ & \\
\hline Rumex crispus $\mathrm{L}$. & $\begin{array}{l}\text { Lengua de } \\
\text { vaca }\end{array}$ & Poligonace ae & & $\mathrm{NC}$ & \\
\hline \multicolumn{6}{|l|}{ Plantas cultivadas } \\
\hline $\begin{array}{l}\text { Saccharum officinarum } \\
\text { L. }\end{array}$ & $\begin{array}{l}\text { Caña de } \\
\text { azúcar }\end{array}$ & Poaceae & $\mathrm{X}$ & $\mathrm{CC}$ & Martín 2004, Evans 2007 \\
\hline Sorghum vulgare Pers. & Sorgo** & Poaceae & $\mathrm{x}$ & $\mathrm{CC}$ & $\begin{array}{l}\text { Russell 1964, Martín } \\
\text { 2004, Evans } 2007\end{array}$ \\
\hline Zea mays $\mathrm{L}$. & Maíz & Poaceae & $\mathrm{x}$ & $\mathrm{CI}$ & $\begin{array}{l}\text { Russell 1964, Martín } \\
\text { 2004,Evans } 2007\end{array}$ \\
\hline
\end{tabular}

* Aceptación: CC: Ciclo completo de la mosca, CI: Ciclo Incompleto de la mosca, NC: No colonizada. ** Plantas que crecieron silvestres en las orillas de cultivos y caminos durante el ciclo agrícola OI06/07 y cultivos establecidos PV-07. 
Distribución de $\boldsymbol{A}$. occiduus en Sinaloa. Periodo 1. Los primeros especímenes de $A$. occiduus se encontraron hacia el norte del estado, entre las ciudades de Guasave y Los Mochis, en las cercanías del ejido Adolfo Ruíz (25 $40^{\circ}$ Latitud Norte, $108^{\circ} 42^{\prime}$ 'Latitud Oeste, $14 \mathrm{msnm}$ ), en un radio $\sim$ de $3.0 \mathrm{~km}$; lo que denota su reciente introducción a esta región. En los muestreos realizados en el Valle del Fuerte sobre los hospederos preferidos la MBC fue localizada en las cercanías del aeropuerto internacional de la ciudad de Los Mochis (25 $41^{\prime}$ Latitud Norte, $109^{\circ} 04^{\prime}$ Latitud Oeste, $4 \mathrm{msnm}$ ) (Fig. 5). Durante diciembre de 2006 se encontraron hojas de zacate Johnson con hasta 500 especímenes (huevos, ninfas y adultos), maíz con más de 700 y caña de azúcar con más de 1000 individuos por hoja en el área del brote. Sin embargo, en los alrededores de las áreas infestadas se encontraron ejemplares de hospederas como zacate Johnson, zacate Bermuda, zacate Buffel y pasto Rhodes con tan sólo nueve individuos en 15 min de búsqueda, (A3). Las densidades disminuyeron gradualmente hasta casi ser imperceptibles a finales de abril de 2007 .

Periodo 2. A partir de mayo de 2007 se realizaron recorridos de campo para confirmar la presencia de la $\mathrm{MBC}$ en las áreas donde previamente había sido detectada. La plaga se registró nuevamente en toda la parte Este del área costera de

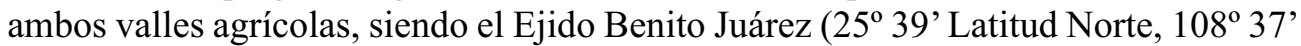
Latitud Oeste, $22 \mathrm{msnm}$ ) el sitio más lejano hacia el sur, el Ejido Cohuibampo (25 $57^{\prime}$ Latitud Norte, $109^{\circ} 10^{\prime}$ Latitud Oeste, $10 \mathrm{msnm}$ ) hacia el norte y el ejido Taxtes ( $25^{\circ} 54^{\prime}$ Latitud Norte, $108^{\circ} 56^{\prime}$ Latitud Oeste, $21 \mathrm{msnm}$ ) hacia el Oeste (Fig.5). En dichas localidades se registraron densidades promedio de cinco especímenes por sitio de colecta, en hospederas como zacate Jhonson, zacate Bermuda, zacate pinto, coquillo, sorgo y caña de azúcar.

Biología, Hábitos y Daños. La mosca blanca de los cereales se alimenta en el envés de las hojas de sus hospederas, aunque algunas veces se le puede encontrar en la vaina de plantas de hojas muy pequeñas (Fig. 4), o en los tallos, cuando es abundante. La hembra oviposita en el envés de las hojas basales de la planta y coloca los huevos de manera individual, desordenados o en grupos de hasta 20 (Fig. 2b). Una vez emergida la ninfa, se desplaza pocos milímetros para fijarse y alimentarse. Después de que la ninfa comienza a alimentarse, pasa por otros tres ínstares ninfales de desarrollo, en cada caso de mayor tamaño, hasta transformarse en el adulto. Al último estadio ninfal comúnmente se le llama "pupa" (Fig. 2c).

Enemigos Naturales. Como parasitoides de la MBC (ninfas) en plantas hospederas, se colectaron algunos representantes de los géneros Encarsia y Eretmocerus (Fig. 6), pertenecientes a la familia Aphelinidae. Estos géneros tienen distribución mundial, y para México se citan nueve especies de Encarsia y tres de Eretmocerus parasitando diferentes especies de moscas blancas, principalmente B. tabaci (Hennessey et al. 1995).

Como depredadores se registraron representantes de Chrysoperla sp, Orius sp, Cycloneda sanguisuga y algunas especies de arañas. 
Vejar-Cota et al. Mosca blanca de los cereales en Sinaloa

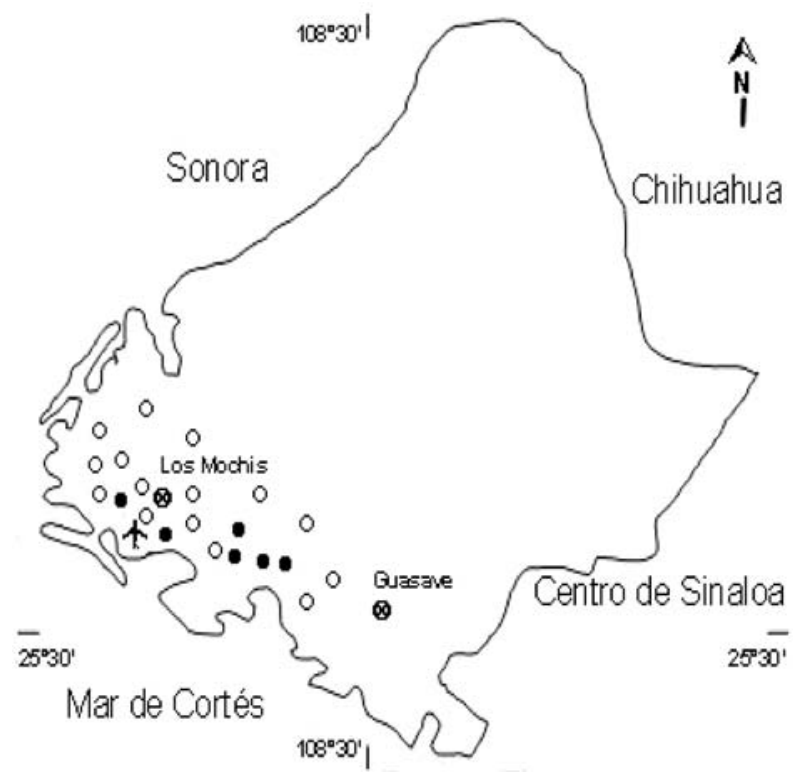

Figura 5. Sitios de distribución de la mosca blanca de los cereales en el norte de Sinaloa. @Ciudad, t Aeropuerto Internacional de la Cd. de Los Mochis, Sitios de colecta del periodo 1 y

Sitios de colecta del periodo 2 .

Impacto Potencial de $A$. occiduus en Gramíneas Cultivadas en el Norte de Sinaloa. En el norte de Sinaloa (Distritos de Guasave y Los Mochis) se siembran $42 \%$ de las gramíneas que se cultivan a nivel estatal (maíz, sorgo, trigo, pastos y caña de azúcar), lo que representa alrededor de 320 mil has entre los ciclos agrícolas otoño-invierno y primavera-verano (INEGI 2001). Del mosaico de gramíneas cultivadas en el estado, el arroz $(O$. sativa) y maicillo ( $S$. bicolor) son los más susceptibles al ataque de $A$ occiduus conocidos a la fecha (Serrano et al. 2006). En Sinaloa se sembraron 1,046 has (arroz) en los Distritos de Culiacán y La Cruz en el ciclo OI-06/07 (SAGARPA, Sinaloa) donde aun no se tiene registro de la presencia de esta plaga. Sin embargo, dentro de las variedades de sorgos se encuentran el forrajero, escobero y granero, los cuales sí están presentes en los Distritos de Guasave y Los Mochis ( $\sim 28$ mil has). Considerando que aun se desconoce el grado de establecimiento que presentará $A$. occiduus en cultivos como trigo y pastos cultivados durante el próximo ciclo agrícola (PV-07) en su actual área de distribución (Fig. 5); es necesario apreciar que ésta podría representar un problema fitosanitario importante en algunos cultivos del norte de Sinaloa, como el sorgo, trigo, pastos, arroz y caña de azúcar, de los cuales se siembran en promedio 60 mil has anualmente. 

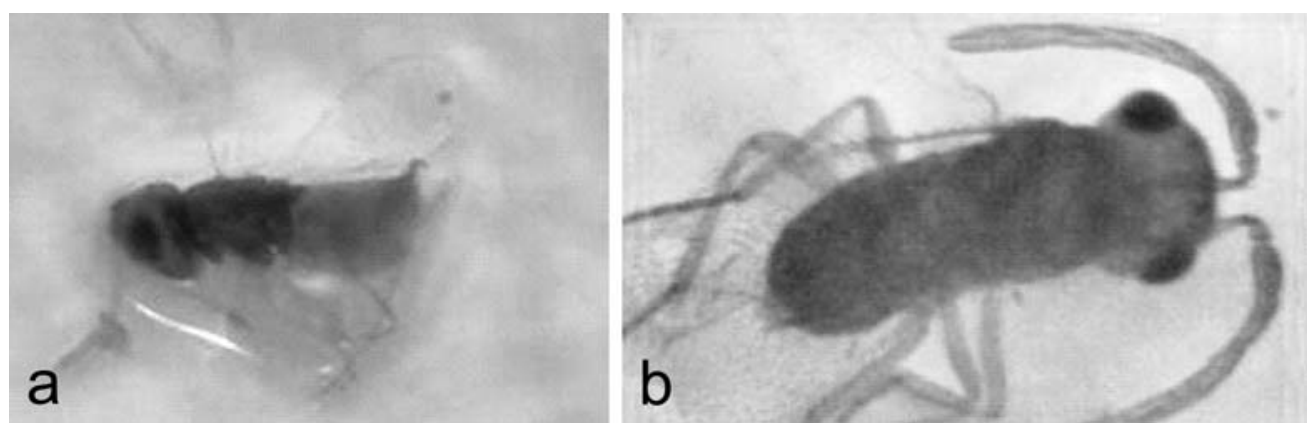

Figura 6. Especies de la familia Aphelinidae parasitando ninfas de A. occiduus en el norte de Sinaloa. a) Encarsia y b) Eretmocerus

\section{DISCUSION}

La mosca blanca de los cereales, $A$. occiduus, es una especie que no había sido detectada en Sinaloa, y dado sus antecedentes en otras regiones de América (Perú y El Salvador), representa un riesgo potencial importante para algunas gramíneas cultivadas y/o sus variedades.

La presencia y reproducción de $A$. occiduus en ocho de las 17 especies vegetales registradas confirma lo documentado por Martín (2004), Serrano et al. (2006) y Evans (2007) referente a que la MBC sólo se reproduce en plantas de hoja angosta, principalmente poáceas y ciperáceas. Es importante señalar que las especies registradas en este estudio como hospederos de la MBC ya habían sido documentadas en diversas referencias, sin embargo, la especie Dichanthium aristatum (Poiret) se cita por primera vez como hospedero potencial de este aleiródido.

Aunque la distribución actual de $A$. occiduus se restringe al norte del estado entre las ciudades de Guasave y Los Mochis en las cercanias del aeropuerto internacional, es probable que el insecto amplie su distribución en el corto plazo debido a la disponibilidad de hospederos y presencia de vientos predominantes de Sur a Oriente (del mar a tierra). Hasta ahora no hay antecedentes de la presencia de esta especie en alguna otra región de México, aunque no se descarta su presencia en Estados vecinos de EUA con registros de $A$. occiduus.

La densidad de población ha sido fluctuante a través del tiempo. En un inicio, durante diciembre de 2006, las poblaciones fueron altas pues se encontraron hojas de zacate Johnson con hasta 500 especímenes (huevos, ninfas y adultos), maíz con más de 700 y caña de azúcar con más de 1000 individuos, sin embargo, durante el invierno de 2007, las poblaciones de la plaga fueron bajas (5 especímenes/ colecta) y a los adultos con frecuencia se les vio volando al nivel del suelo, o reposando en las hojas de sus hospederas. 
El ciclo biológico y daños causados por $A$. occiduus son practicamente similares a otros aleiródidos. Serrano et al. (2006) mencionan que el ciclo de vida de $A$. occiduus en arroz, de huevo a adulto, puede completarse en dos a tres semanas; pero su duración puede variar en función de la temperatura, humedad y hospedera. Poinar (1965) anota que el ciclo transcurre en 36 a 50 días en Cyperus rotundus. La alta incidencia de la MBC, en países como Perú (1999) y El Salvador (2003), ha ocasionado pérdidas parciales o totales en cultivos de arroz y sorgo. El daño principal lo causan las ninfas y adultos durante su alimentación, al detener el desarrollo de la planta (amarillamiento) y cubrir de fumagina las hojas inferiores, lo que ocasiona la muerte de la planta y/o la infertilidad del fruto (Serrano et al. 2006). Sin embargo, la presencia de $A$. occiduus durante el ciclo agrícola OI-06/07, en el norte de Sinaloa, no ocasionó daños importantes, lo cual pudo deberse entre otros factores a la presencia de enemigos naturales, ausencia de hospederas susceptibles o condiciones climáticas desfavorables para el desarrollo de la plaga. Sin embargo, en el ciclo agrícola PV-07, ya se detectó la presencia de huevos y ninfas en sus principales hospederas, tanto cultivadas (sorgo y caña de azúcar) como silvestres.

Como parasitoides se registraron algunos individuos de la familia Aphelinidae con baja actividad párasitica, sin embargo, dicho hallazgo es muy importante puesto que de las especies de afelínidos (30 especies) citadas mundialmente, sólo se reportan a Encarsia luteola Howard y E. deserti Rivnay \& Gerling, parasitando a A. occiduus (Gerling 1967, Martín 2004), por lo que las especies encontradas en el norte de Sinaloa podrían ser nuevos registros. Además de las especies de afelínidos, Evans (2007) cita a Euderomphale hyalina (Compere \& Annecke) de la familia Eulophidae parasitando a la MBC.

Basados en las observaciones realizadas en el área con presencia de $A$. occiduus, en el maíz, principal cultivo que se siembra en Sinaloa, no se vislumbra, en el corto plazo, ningún riesgo potencial en las variedades sembradas en el ciclo agrícola OI06/07 (Asgrow: Bisonte, Puma y Sable; Pionner: 30P45, 30M16, 30P49, 30G59, 30G57 y 30654), ya que las ninfas no alcanzan su desarrollo en este cultivo, y por ende no puede ocasionar pérdidas económicas. Durante los muestreos se observaron hasta 700 huevos de la mosca en las hojas basales de este cultivo, sin embargo, ninguna ninfa logró alcanzar la madurez. Por otro lado, cultivos como sorgo, trigo, pastos y caña de azúcar (o sus variedades), podrían ser atacados y ocasionar daños importantes en $\sim 60$ mil has que se siembran en el norte de Sinaloa conforme se vaya dispersando y expresando esta plaga, durante el ciclo agrícola PV-07 y OI-07/08.

Aunque el área actual de la MBC se restringe al norte del municipio de Guasave y alrededores del Aeropuerto Internacional de la Ciudad de Los Mochis, es imprescindible ampliar los muestreos en el Valle del Fuerte y otras regiones de Sinaloa, ya que la dispersión de este tipo de insectos se facilita con la disponibilidad de hospederos y vientos fuertes, por lo que es probable amplíe su distribución en el corto plazo. 
El presente estudio aporta los primeros conocimientos sobre $A$. occiduus en Sinaloa, México y brinda importantes elementos para iniciar programas de manejo de la plaga. Dentro de las medidas de manejo y cuidados que se deben tener para reducir el impacto de la $\mathrm{MBC}$, se recomienda: a) evitar la movilización del insecto a los valles de Sinaloa donde aún se siembra el arroz, por ser un cultivo susceptible; b) mantener constante vigilancia en los cultivos susceptibles para detectar en forma oportuna la presencia de la mosca blanca; c) limitar el desarrollo de hospederas silvestres en los alrededores de cultivares susceptibles una vez detectada la presencia de la MBC; d) mantener el monitoreo constante de la MBC para detectar su dispersión e incrementos poblacionales; e) utilizar métodos de control alternativos a los químicos para mantener la acción de los parasitoides y otros enemigos naturales; e) limitar, en caso de aplicación de insecticidas, el uso de productos y dosis que promuevan el desarrollo de resistencia; y f) aplicar las medidas fitosanitarias establecidas para el manejo de otras especies de moscas blancas en el norte del estado.

AGRADECIMIENTOS. Se agradece a la Cía. Azucarera de Los Mochis, S. A de C. V. y Agrícola Ohuira, S.A. de C. V. por la oportunidad de dar a conocer esta información a la comunidad agrícola regional, al Ing. Adolfo Caro Gastelum por proporcionar los primeros especímenes para la determinación de la MBC, al M.C. Jorge Valdez Carrasco por la asistencia durante el trabajo fotográfico, al T. A. Reynaldo Armenta Aguilar por el apoyo técnico y logístico en los muestreos realizados en el norte de Sinaloa y a los Ings. Leopoldo Serrano Cervantes y Reyna F. Guzmán de Serrano por su apoyo con literatura y comentarios sobre esta plaga en el Salvador.

\section{LITERATURA CITADA}

Anónimo. 2003. Fundación Produce. Guía para la asistencia técnica agrícola para el área de influencia del campo experimental valle del Fuerte. Sexta Edición. Sinaloa, México. 208 p.

Evans, G.A. 2007. The whiteflies (Hemiptera: Aleyrodidae) of the world and their host plants and natural enemies. USDA/APHIS. (Disponible en: http://www.sel.barc.usda.gov) (Revisado el 15 marzo de 2007).

Gerling, D. 1967. Bionomics of the whitefly parasites complex associated with cotton in southern California (Homoptera: Aleyrodidae; Hymenoptera: Aphelinidae). Annals of the Entomological Society of America 60: 1306-1321.

Heinrichs, E. A.\& A.T. Barrion. 2004. Rice-feeding insects and selected natural enemies in West Africa: biology, ecology, identification. Los Baños (Philippines): International Rice Research Institute and Abidjan (Côte d'Ivoire): WARDA-The Africa Rice Center. 243 p.

Hennessey, R. D., H. C. Arredondo-Bernal \& L. A. Rodríguez-Del-Bosque. 1995. Distribución geográfica y huéspedes alternos de parasitoides afelínidos de Bemisia tabaci (Homoptera: Aleyrodidae). Vedalia 2: 61-75.

Hodges, G. S.\& G.A. Evans. 2005. An identification guide to the whiteflies (Hemiptera: Aleyrodidae) of the Southeastern United States. Florida Entomologist 88 (4): 518-534.

INEGI. 2001. Anuario estadístico Sinaloa. Gobierno del Estado de Sinaloa. 524 p.

Martín, J.H. 1987. An identification guide to common whitefly pest species of the World (Homoptera: Aleyrodidae). Tropical Pest Management 33:298-322. 
Martín, J.H. 2004. Whiteflies of Belize (Hemiptera: Aleyrodidae). Part 1. Introduction and Account of the Subfamily Aleurodicinae Quaintance \& Baker. Zootaxa 681: 1-119.

Martin, J.H. \& L.A. Mound. 2007. An annotated check list of the world's whiteflies (Insecta: Hemiptera: Aleyrodidae). Zootaxa 1492: 1-84.

Ortega A., L.D. 1995. Colecta, Montaje y Determinación de Aleyrodidae. Fitófilo 48 (88): 53-70.

Ortega A., L.D. 1999. Mosca blanca vectora de virus en hortalizas (Homoptera: Aleyrodidae) pp: 149176. In: S. Anaya R. y J. Romero N. (eds.). Hortalizas, plagas y enfermedades. Editorial Trillas. México D. F. México.

Poinar, G.O. 1965. Observations on the biology and ovipositional habits of Aleurocybotus occiduus (Homoptera: Aleyrodidae) attacking grasses and sedges. Annals of the Entomological Society of America 58 (5): 618-620.

Russell, L.M. 1964. A new species of Aleurocybotus (Homoptera: Aleyrodidae). Proceedings of the Entomological Society of Washington 66 (2): 101-102.

Russell, L.M. 2000. Notes on the family Aleyrodidae and its subfamilies: redescription of the genus Aleurocybotus Quaintance and Baker and description of Vasdavidius, a new genus (Homoptera: Aleyrodidae). Proceedings of the Entomological Society of Washington 102: 374-383.

Serrano, C.L., R.F. Guzmán S., C.A. Borja M., A.E. Moran, A. Lemus M., \& E. Castillo M. 2004. Plaga de moscas blancas (Aleurocybotus occiduus) infestando arroz (Oryza sativa) y maicillo (Sorghum bicolor), en El Salvador, América Central: 2003. Memoria del IX Congreso Internacional de Manejo Integrado de Plagas. El Salvador, Centroamértica. 3-5 nov. 2004. 96 p.

Serrano, C.L., R.F. Guzmán S., C.A. Borja M., A.E. Moran, A. Lemus M., \& H. Pitre. 2006. Plaga de mosca blanca (Aleurocybotus occiduus) en arroz (Oryza sativa) y maicillo (Sorghum bicolor), El Salvador, América Central: 2003-2005. Memoria del 52 Reunión Anual del Programa Cooperativo Centroamericano para el Mejoramiento de Cultivos y Animales (PCCMCA). Montelimar, Nicaragua.

Quaintance, A.L.\& A.C. Baker. 1914. Classification of the Aleyrodidae Part II, Tech. Series, US Department of Agriculture Bureau of Entomology 27(2): 95-109.

Recibido: 14 de septiembre de 2007

Aceptado: 8 de octubre de 2008 